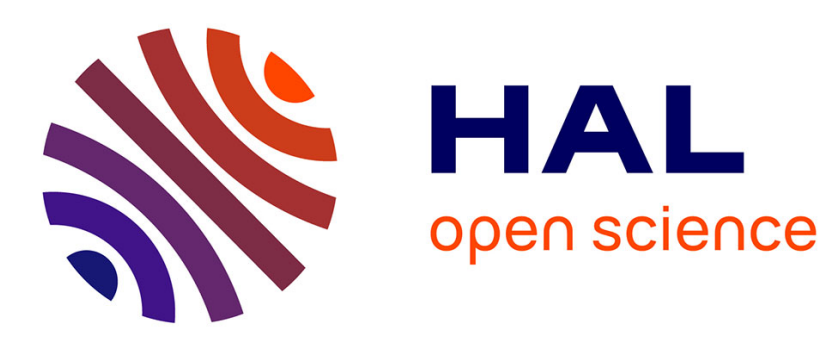

\title{
Enhancing EEG Surface Resolution by Using a Combination of Kalman Filter and Interpolation method
}

Ibtissem Khouaja, Ibtihel Nouira, Mohamed Hedi Bedoui, Mohamed Akil

\section{To cite this version:}

Ibtissem Khouaja, Ibtihel Nouira, Mohamed Hedi Bedoui, Mohamed Akil. Enhancing EEG Surface Resolution by Using a Combination of Kalman Filter and Interpolation method . 13th Computer Graphics, Imaging and Visualization, Mar 2016, Beni Mellal, Morocco. hal-01801279

\section{HAL Id: hal-01801279 \\ https://hal.science/hal-01801279}

Submitted on 28 May 2018

HAL is a multi-disciplinary open access archive for the deposit and dissemination of scientific research documents, whether they are published or not. The documents may come from teaching and research institutions in France or abroad, or from public or private research centers.
L'archive ouverte pluridisciplinaire HAL, est destinée au dépôt et à la diffusion de documents scientifiques de niveau recherche, publiés ou non, émanant des établissements d'enseignement et de recherche français ou étrangers, des laboratoires publics ou privés. 


\section{Enhancing EEG Surface Resolution by Using a Combination of Kalman Filter}

\section{and Interpolation method}

\author{
Ibtissem KHOUAJA, Ibtihel NOUIRA, M. Hedi BEDOUI \\ Laboratory of Medical Imaging and Technology \\ Faculty of Medicine \\ Monastir, Tunisia \\ khouaja.ibtissem@gmail.com \\ btihelnouira@gmail.com \\ medhedi.bedoui@fmm.rnu.tn
}

\author{
Mohamed AKIL \\ Laboratory of Computer science (LIGM), ESIEE-Paris \\ University of Paris-Est Marne-La-Vallée \\ Paris, France \\ mohamed.akil@esiee.fr
}

\begin{abstract}
With recent progress in the medical signals processing, the EEG allows to study the Brain functioning with a high temporal and spatial resolution. This approach is possible by combining the standard processing algorithms of cortical brain waves with characterization and interpolation methods. First, a new vector of characteristics for each EEG channel was introduced using the Extended Kalman filter (EKF). Next, the spherical spline interpolation technique was applied in order to rebuild other vectors corresponding to virtual electrodes. The temporal variation of these vectors was restored by applying the EKF. Finally, the accuracy of the method has been estimated by calculating the error between the actual and interpolated signal after passing by the characterization method with the Root Mean Square Error algorithm (RMSE).
\end{abstract}

Keywords-EEG, Extended Kalman Filter, Signal characterization, Spherical Spline Interpolation, Root Mean Square Error.

\section{INTRODUCTION}

The Electroencephalogram (EEG) provides information in real time with a very high temporal resolution in the order of 10 milliseconds, albeit with a poorer spatial resolution in the order of few millimeters to one centimeter. It is the registration of the sensorimotor cortex rhythms by using implanted electrodes in invasive manner, or by using non invasive methods from scalp [1]. EEG is a routine clinical procedure, widely used in the monitoring and quantification of the levels of drowsiness and awakening. It allows also recognizing abnormalities according to the potential and frequencies aspects [2]. The signals recorded by the EEG are a mixture of different sources that produce the electrical activity in the brain. Generally, the number of sources is much higher than the number of sensors. For this reason, it is interesting to increase the number of sensors. In this context, many works were conducted. Three dimensional interpolation's algorithm like the 3D polynomial interpolation and the spherical polynomial interpolation are used in [3] and [4]. Nevertheless these methods gave unsatisfactory results, especially in case of the smallest orders. In [5], the authors suggest seven interpolation methods as barycentric, polynomial and non linear spline interpolation. However, these studies were suffered of the high error of interpolation. The result indicates that the optimal model for the interpolation of the cortical activity is a 2D-spline.

The aim of this communication is to enhance the spatial resolution of EEG. The idea is to start from a reduced number of time-varying data collected at the surface to a distribution in different points not covered by sensors. The estimation of signal parameters follows the multivariate autoregressive (MVAR) modeling of EEG time series. The estimated MVAR parameters are interpolated and transformed to the temporal domain to describe new timevarying signals. Among all MVAR estimation approaches, a Kalman filter based MVAR modeling gained wider applications due to its accurate and high dimentional EEG data [6], [7], [8]. The MVAR model analysis explains the spectral composition of the signal and generates in the adaptive way a vector of parameters describing the evolutionary spectrum. First, the EKF is a data assimilation method permitting the state modeling. Second the spherical spline method is known to be particularly robust in the EEG signal interpolation. The combination with a 2D-Spline algorithm allows an efficient interpolation result.

The paper is organized as follow: Section 2 describes the data used in this approach and represents the methodology based on windowing, AR modeling, vectors interpolation and validation of the different step by calculating the RMSE error. In section 3, the result of these methods on simulated data are presented and discussed.

\section{METHODOLOGY}

A bio-mathematical model was developed on two axes. In the first axis, EEG signal was decomposed into windows. Then, the EKF was applied to extract features from each window. An autoregressive (AR) vector of characteristics parameters evolving in the time and describing the dynamicity of the signal in the time domain, was been estimated. The second axis presents a 3D interpolation technique to improve the spatial resolution of the EEG. Our physiological knowledge was been integrated in models selection, also, in the interpolation of the signals situated in the same lobe. 


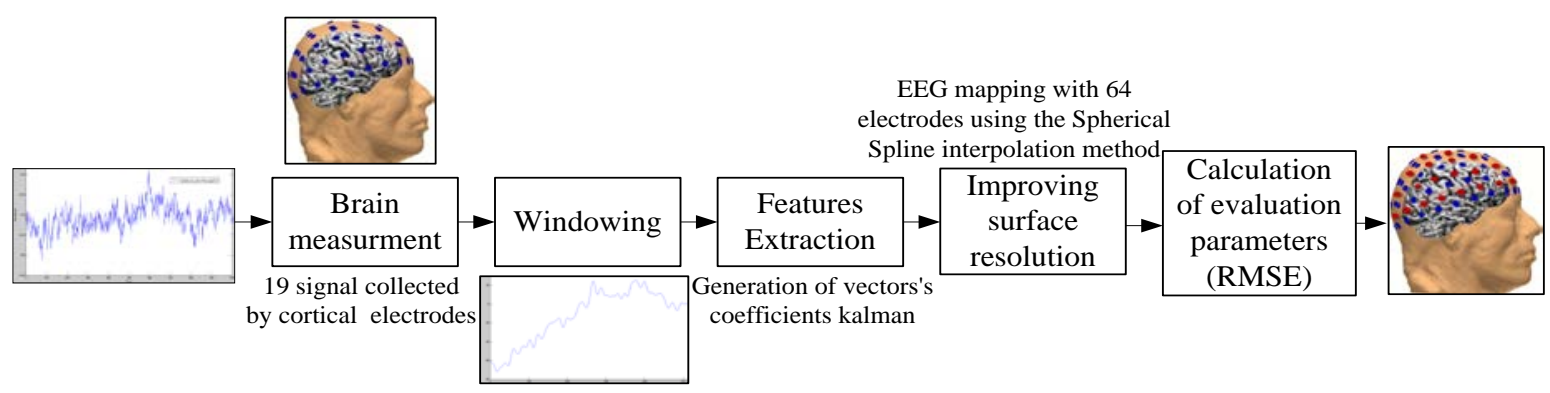

Figure 1. Bloc diagram of modeling and improvement surface resolution of scalp EEG

The RMSE was calculated in the first time between the actual and the reconstructed signal from the AR vector. In the second time, the RMSE was calculated between the actual and the reconstructed signal from the interpolated $\mathrm{AR}$ vector.

\section{A. The data set}

FirstThe data used in our study was obtained from the online international data set recorded according to the 10/20 standard (http://physionet.org/pn4/eegmmidb/). Eleven subjects are participated. The length of recorded 1signal was four minutes with a sampling rate $512 \mathrm{~Hz} .64$ signal situated in the different cortical lobe for each subject, are used in the validation step

\section{B. Features Extraction}

The electrical activity of the brain is usually divided into three categories: the bioelectrical events produced by the simple neurons, evoked potentials (EP) and the spontaneous activity. The spontaneous activity is measured on the scalp or on the brain and is collected in the form of a signal highly variable in the time. The electrical activities rhythmic brain was classified according to their frequencies in the band of the $0.1 \mathrm{~Hz}$ to $100 \mathrm{~Hz}$ [9]. The spontaneous EEG is characterized as a linear stochastic process presenting similarities with noise. It is a pseudo-random signal, easily affected by various physiological noise (ECG, EOG, and EMG) and electrical artifacts. The signal also shows a high degree of non-stationary. The EEG, being a continuous signal in the time, is individualized in period (Figure 2) in order to facilitate its analysis.

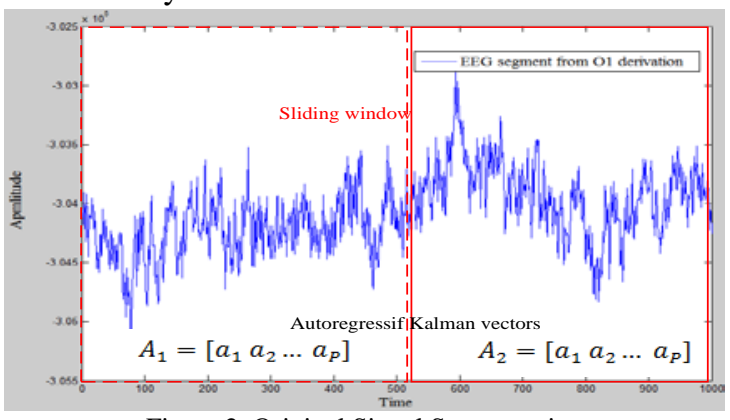

Figure 2. Original Signal Segmentation
This time period is called sampling window or "time". For a random non periodic signal such is the case of the EEG, it has selected a time of a very short duration. For example, during a second the EEG signal does not change of pace. The duration of a second can be considered as a period [10].

Minimize the amount of information helps to reduce the complexity of the signal and to better understand its dynamics variation. The modeling of the EEG signals part of a model auto-regressive at a modeling by a set of coefficients [11]. This is the estimate of new parametric vectors representing the dynamic variation of the EEG signal. Typically, the estimation of these parameters autoregressive is performed using a Kalman filter selfadaptive [12] [2]. However, it is demonstrated that this may represent a erroneous modeling a view that the EEG signal undergoes changes instantaneous. The Kalman filter is an adaptive process that updates the initial estimates of the coefficients in the Model AR in function of each new observation of the signal [12]. The update is proportional to the difference between the new observations and the predicted values of the coefficients. Although, the performance of this model depends on two parameters, the first is the filter order, the second is entry window width. The optimal order of the model can be estimated by using different methods such as the AIC (Akaike's Information Criterion) [6] or the SBC (Shwartz's baysian criterions) [6]. For models ranging in time, the best order is estimated using SBC. Its value is maintained constant during the process.

The reconstruction of the state in terms of the measures is then a problem of Bayesian estimation that determines the law of probability to retrospectively in the light of available measures [13] [19]. A multivariate autoregressive (MVAR) model combined with Extended Kalman filter has been used in the neurophysiological signal analysis. In this context, many works are concucted as [6], [14], [15] and [18]. In these studies, the MVAR model represents the time-varying with a high precision.

To use the Extended Kalman filter, there is a need to establish the model space-state for the observations. The multivariate autoregressive (MVAR) model defined as: [8]: 
$\left[\begin{array}{c}y_{t 1}(n) \\ \vdots \\ y_{t N}(n)\end{array}\right]=\sum_{r=1}^{p} A_{r}(n)\left[\begin{array}{c}y_{t 1}(n-r) \\ \vdots \\ y_{t N}(n-r)\end{array}\right]+\left[\begin{array}{c}w_{1}(n) \\ \vdots \\ w_{N}(n)\end{array}\right]$

With $y_{t(1: N)}$ is the time series, $r=1: P$, with $\mathrm{P}$ is the optimal order of the model, $w_{(1: N)}$ is the White Gaussian Noise due to the observation or measurement), $A_{t}^{(i)}$ is the scalable parameter which corresponds to a matrix of AR coefficients defines by :

$A_{r}(n)=\left[\begin{array}{ccc}a_{11}^{r}(n) & \ldots & a_{11}^{r}(n) \\ \vdots & \ddots & \vdots \\ a_{11}^{r}(n) & \ldots & a_{11}^{r}(n)\end{array}\right]$

The optimum combination for features extraction with Adaptive EKF will be used as a procedure for reduction of EEG channels and enhancing the quality of information collected from the cortex.

\section{Interpolation Method}

In order to reconstruct new cortical signals, spherical spline interpolation was performed using the Perrin and al. algorithm [16]. The algorithm receives the 3D currently connected electrodes coordinates $(\mathrm{x}, \mathrm{y}, \mathrm{z})$ and $\mathrm{AR}$ vectors describing the temporal variation of each signal. The distance between 3D electrodes is calculated as in [5].

Let us denote:

- Es is a set of $M$ real points $e s_{l}(l=1 \ldots M)$ where real potential valueVs $s_{1}$ was measured.

- $E$ is a set of $\mathrm{N}$ virtual points $e_{k}(\mathrm{k}=1 \ldots \mathrm{N})$ where interpolated cerebral activity $V_{k}$ was calculated and $\left(x_{k}, y_{k}, z_{k}\right)$ was respectively, the $e s_{l}$ and the $e_{k}$ point coordinates.

The spherical spline interpolation assumes that the cerebral activity $V_{k}$ at any point $e_{k}$ can be defined by the following equation:

$$
V_{k}=c_{0}+\sum_{l=1}^{M} c_{l} \cdot g\left(\cos \left(e_{k}, e s_{l}\right)\right)
$$

Where $c_{l}(l=1 \cdots M)$ was $C$ vector coefficients which are determined by the following linear equations:

$$
G c+t c_{0}=V s
$$

Let us denote:

$$
\begin{aligned}
& t=[1,1, \ldots, 1]^{t} \\
& G=\left[g_{i j}\right]=g\left(\cos \left(e_{s i}, e_{s j}\right)\right)
\end{aligned}
$$

The function $\mathrm{g}$ is calculated by the $\mathrm{Eq}(8)$ :

$g(x)=\frac{1}{4 \pi} \cdot \sum_{n=1}^{\infty} \frac{2 n+1}{n^{m}(n+1)^{m}} \cdot P_{n}(x)$

$\mathrm{m}$ is the order of the $V_{s}$ spline, $P_{n}(x)$ is the ordinary Legendre polynomials of order $\mathrm{n}$ which have been calculated recursively as follows:

$$
\left\{\begin{array}{c}
P_{0}(z)=1 \\
P_{1}(z)=z \\
P_{l+1(z)}=\frac{1}{2 l+1}\left[(2 l+1) P_{l}(z)-l P_{l-1}(z)\right]
\end{array}\right.
$$

All distances separating the electrodes are provided with recording's files. From 19 actual points, 64 points are generated by our approach. The difference between the simulated and actual results is calculated for each new simulated point by the RMS Error. This result is summarized in the TABLE I.

\section{RMS Error}

To compare the reconstructed signal with the real many researchers use the Root Mean Square Error (RMSE) as measure of quality because it is relatively easy to analyze. The RMSE calculates the error between the real values and the interpolated ones. For comparing the quality of the different interpolation methods, it is important that the matching between the measured values and their corresponding interpolated cerebral values should be compared. This matching is judged by computing the RMSE.

The RMSE is calculated as follow [17]:

$$
R M S E=\frac{\|V r-V\|}{\|V r\|}
$$

With : The superscript \|\| denoting the Euclidean norm, $V r$ is a vector of the 64 measured values, and $V$ is the corresponding interpolated values.

\section{RESULTS AND DISCUSSION}

The problem discussed in this communication is the EEG capability increase the information collected to provide a large number of information from sensors collected on the surface of the cerebral cortex via a limited number of electrodes. The proposed method, simple to implement and relevant, is to provide an interpolation algorithm, based on the spherical spline, vectors, auto-regressive representing the temporal variation of the signals at each moment in time. The improvement is demonstrated in a simulation framework on 64 electrodes which 19 are actually connected.

The goal of EEG signal parameterization is to minimize the amount of information contained in a signal without change the medical signification of the original signal. Therefore, the EEG signal was decomposed into short segment of one second. In this case, the signal was considered periodic and stationary. The EKF filter was applied to each segment and generated AR vectors of 10 coefficients. From these vectors, their temporal variation was reconstructed with the same characteristics.

Then, EEG signal was interpolated by 3D-spline as follow [17] The simulation was tested using MATLAB on a laptop giving Core ${ }^{\mathrm{TM}} \mathrm{i} 3-2310 \mathrm{CPU}$ with a frequency of 
$2.10 \mathrm{GHz}$ and RAM of $4.00 \mathrm{GO}$. Tab 1 presents a comparative study of five signals issue from electrodes actually collected on the cortex.

TABLE I. EVALUATION RESULTS OF RECONSTRUCTION SIGNALS

\begin{tabular}{|c|c|c|c|c|c|}
\hline Reconstructed & \multicolumn{5}{|c|}{ Signal's } \\
\cline { 2 - 6 } Signal : & C2 & CP3 & F6 & Fc5 & PO7 \\
\hline $\begin{array}{c}\text { From 3D- } \\
\text { Spline } \\
\text { interpolation }\end{array}$ & 0.22 & 0.003 & 0.1577 & 0.552 & 0.008 \\
\hline $\begin{array}{c}\text { From 3D- } \\
\text { spline } \\
\begin{array}{c}\text { Interpolation } \\
\text { of AR Kalman } \\
\text { vectors }\end{array}\end{array}$ & 0.0288 & 0.0531 & 0.0462 & 0.0488 & 0.0327 \\
\hline
\end{tabular}

The spline family is characterized by the most minimal RMS error mean; in particular the 3D-spline interpolation. Moreover, the combination with Kalman filter generates the best reconstruction and offers the closest interpolated signal to the real EEG as shown in Figure 3.

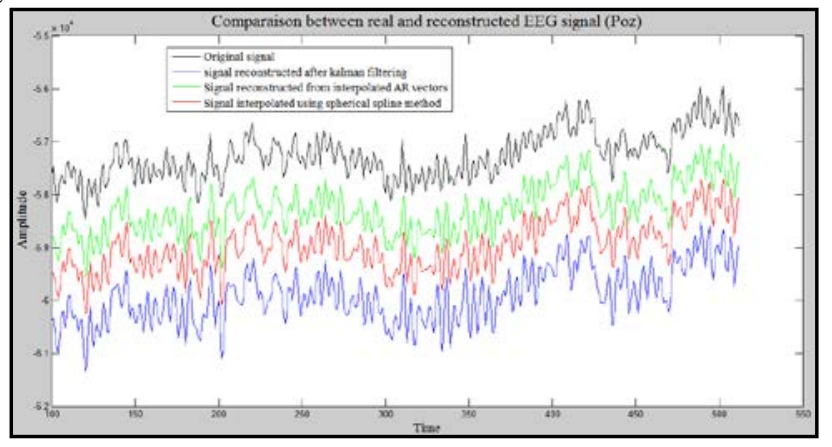

Figure 1. Temporal variation of real and interpolated EEG signal

Figure 3 illustrates the comparaison between the real and the interpolated EEG time varying of the Po7electrode of a normal patient.

TABLE 2 represents the RMS error, made upon 11 healthy subject's EEG records in the different neurosensory conditions. The Error was calculated between the real EEG and the reconstructed one on 64 signals for each patient.

TABLE II. GLOBAL EVALUATION OF THE METHODOLOGY

\begin{tabular}{|c|c|}
\hline Reconstruction Method & RMSE \\
\hline $\begin{array}{c}\text { Temporal reconstruction using 2D- } \\
\text { Spline interpolation }\end{array}$ & 0.1163 \\
\hline $\begin{array}{c}\text { Temporal reconstruction using EKF } \\
\text { coefficient's vector }\end{array}$ & 0.0643 \\
\hline $\begin{array}{c}\text { Temporal reconstruction using coupling } \\
\text { between coefficient's vector and 2D- } \\
\text { Spline interpolation }\end{array}$ & 0.0417 \\
\hline
\end{tabular}

The biomedical signals like EEG are characterized by a high sensibility, and a strong dynamic aspect. The result showen in TABLE 2 demonstrate that the RMS error evaluating the time-varying reconstruction of 11 patients, by different methods, was the smallest by the approach combining the AR Kalman filter with the 3D-Spline interpolation algorithm.

\section{REFERENCES}

[1] Lotte, F, Congedo, M, Lercuyer, A, et al. A review of classification algorithms for EEG-based brain-computer interfaces. Journal of neural engineering, 2007, vol. 4.

[2] 3Oikonomou, V. P., Tzallas, A. T., Tsalikakis, D. G., Fotiadis, D. I., \&Konitsiotis, S. (2009). "The Use of Kalman Filter in Biomedical Signal Processing”. INTECH Open Access Publisher.

[3] Kang, Seung Suk, Timothy J. Lano, and Scott R. Sponheim. "Distortions in EEG interregional phase synchrony by spherical spline interpolation: causes and remedies." Neuropsychiatric Electrophysiology 1.1 (2015): 1.

[4] Ciulla, Carlo, et al. "A compilation on the contribution of the classic-curvature and the intensity-curvature functional to the study of healthy and pathological MRI of the human brain." International Journal of Applied Pattern Recognition 2.3 (2015): 213-234.

[5] Soufflet. L, Toissaint. M, Lurthringer. R and al. "A Statistical Evaluation of the Main Interpolation Methods Applied to3Dimentional EEG Mapping”, In Electroencephalography and clinical neurophysiology, Elsevier scientific publishers Ireland, (1991) pp. 393-402.

[6] Omidvarnia, A. H., Mesbah, M., Khlif, M. S., O'Toole, J. M., Colditz, P. B., \&Boashash, B. (2011, August). "Kalman filterbased time-varying cortical connectivity analysis of newborn EEG”. In Engineering in Medicine and Biology Society, EMBC, 2011 Annual International Conference of the IEEE (pp. 14231426). IEEE.

[7] Garg, Harish Kumar, and Amit Kumar Kohli. "EEG spike detection technique using output correlation method: A Kalman filtering approach." Circuits, Systems, and Signal Processing 34.8 (2015): 2643-2665.

[8] Ghumare, Eshwar, et al. "Comparison of different Kalman filter approaches in deriving time varying connectivity from EEG data." Engineering in Medicine and Biology Society (EMBC), 2015 37th Annual International Conference of the IEEE. IEEE, 2015

[9] Niedermeyer. E, Lopes da Silva. F. H. "Electroencephalography: Basic Principles, Clinical Applications, and Related Fields”, Lippincott Williams \& Wilkins, 2005 - 1309 pages

[10] Jansen, B. H., Bourne, J. R., \& Ward, J. W. (1981). "Autoregressive estimation of short segment spectra for computerized EEG analysis". Biomedical Engineering, IEEE Transactions on, (9), 630-638.

[11] F. Wendling, F. Bartolemei, J. J. Belanger, and al, F. Wendling, F. Bartolemei, J.J. Bellanger, P.Chauvel. Epileptic fast activity can be explained by a model of impaired GABAergic dendritic inhibition. Européen Journal of Neuroscience, Vol15, pp. 1499$1508,2002$.

[12] A. SAYDATUL, PAULRAJ. MP, SAZALI Y. (2011) "Analysis of EEG signals during relaxation and mental stress condition using AR modeling techniques", In IEEE International Conference on Control System, Computing and Engineering.

[13] Barton, M. J., Robinson, P., Kumar, S., Galka, A., DurrantWhyte, H. F., Guivant, J., \& Ozaki, T. (2009). "Evaluating the performance of kalman-filter-based eeg source localization”.Biomedical Engineering, IEEE Transactions on, 56(1), 122-136.

[14] L. Astolfi, F. Cincotti, D. Mattia, F. De Vico, A. Tocci, A. Colosimo, S. Salinari, M. G. Marciani, W. Hesse, H. Witte, M. Ursino, M. Zavaglia, and F. Babiloni, "Tracking the Time- 
Varying Cortical Connectivity Patterns by Adaptive Multivariate Estimators," IEEE Trans. Biomed. Eng., vol. 55, pp. 902-913

[15] L. Faes, S. Erla, and G. Nollo, "Measuring Connectivity in Linear Multivariate Processes: Definitions, Interpretation, and Practical Analysis," Comput. Math. Methods Med., vol. 2012, p. 18, 2012.

[16] J. J. BELLANGER, D. OMRANE, F. WENDLING, (2003) 'Modélisation et identification de modèles neuronaux pour les signaux EEG en épilepsie'. In $19^{\circ}$ Colloque sur le traitement du signal et des images, FRA, GRETSI.

[17] IbtihelNouira, Asma Ben Abdallah, SihamLayouni, Mohamed HédiBedoui and Mohamed Dogui, "Spectral density variation mapping of cerebral waves by Three-Dimensional interpolation techniques ”, International Journal of Imaging Systems and Technology, Vol. 25, pp. 191-198, September 2015.

[18] L. Faes and G. Nollo, "Extended causal modeling to assess Partial Directed Coherence in multiple time series with significant instantaneous interactions," Biol. Cybern., vol. 103, pp. 387-400, 2010/11/01/ 2010.

[19] Omidvarnia, A. H., Atry, F., Setarehdan, S. K., \&Arabi, B. N. (2005, September). "Kalman filter parameters as a new EEG feature vector for BCI applications". In Signal Processing Conference,.13th European (pp.1-4). IEEE. 